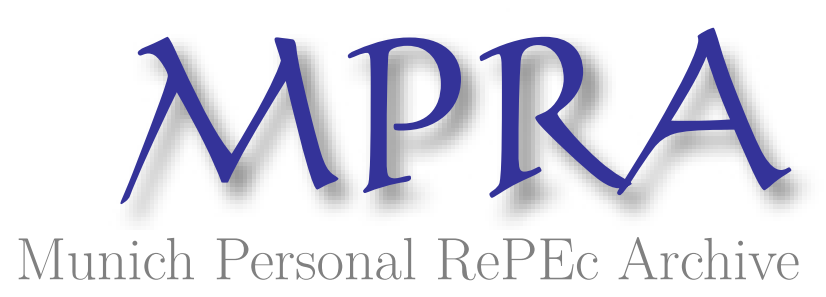

\title{
The roles of reputation and transparency on the behavior of biased experts
}

Bourjade, Sylvain and Jullien, Bruno

2011

Online at https://mpra.ub.uni-muenchen.de/34813/

MPRA Paper No. 34813, posted 17 Nov 2011 17:24 UTC 


\title{
The Roles of Reputation and Transparency on the Behavior of Biased Experts*
}

\author{
Sylvain Bourjade ${ }^{* *} \quad$ Bruno Jullien***
}

November 2010

\begin{abstract}
We analyze situations in which an expert is biased toward some decision but cares also about his reputation in the market for experts. The information the expert reveals decreases as his bias moves toward stronger preference for the status quo. We show that it is optimal to publicly disclose both the expert's contribution and his identity. Surprisingly, revealing the intensity of the expert's bias doesn't always improve the information he reveals in equilibrium. The presence of a second expert raises the first expert's incentives to report truthfully when reports are public, but reduces them when they are secret. In particular, having an option to call another expert may be detrimental in terms of information production if reports are not public. Finally, sequential consultation of experts reduces the information obtained when reports are public, but raises it when they are secret.
\end{abstract}

Keywords: Experts, Bias, Reputation.

JEL Classification: D82, L40.

${ }^{*}$ We thank participants to the seminars at Universitat Autonoma de Barcelona, Université Paris I, Université Lyon II, Université Paris Ouest - Nanterre and Toulouse Business School, the IIOC 2005 conference in Atlanta, the 6th Journées Louis-André Gérard-Varet in Marseille, the EARIE 2008 Conference in Toulouse, the EEA-ESEM 2009 conference in Barcelona and the JEI 2010 in Madrid for helpful comments. Benjamin Hermalin suggested the title; the previous title was "Expertise and Bias in Decision Making." This work was partially written while the first author was a Marie Curie fellow at Universitat Autonoma de Barcelona. All remaining errors are ours.

**Toulouse Business School. E-mail: s.bourjade@esc-toulouse.fr

***Toulouse School of Economics (IDEI and GREMAQ). E-mail: bjullien@cict.fr 


\section{Introduction}

In many economic environments, the information critical for decision making is not readily available, but, instead, obtained from experts. A key issue is that experts could be biased, perhaps because they have a financial stake in the ultimate decision.

For instance, the mission of the U.S. Food and Drug Administration (FDA) is to monitor and control the quality of health products. FDA's decisions are motivated through the advice of advisory committees providing "independent expertise and technical assistance related to the development and evaluation of products regulated by FDA. ${ }^{1}$ " Most advisory committee members are expert scientists who are often consultants for both the FDA and regulated industry. There has been an increasing interest in the financial conflicts of interest of FDA advisory committee members such as those for Vioxx, Levaquin, and Tysabri. (See, for instance, Angell, 2005; Cauchon, 2000; Harris, 2006; Henderson, 2006.) To reduce those conflicts of interests, committee members have to complete a Confidential Financial Disclosure Report on which they must disclose current and past financial interests related to the current case. Waivers may then be granted when the member's expertise is more valuable than the potential for a conflict of interest.

Similarly, when evaluating a merger the Competition Authority must elicit the relevant information from experts, often biased in favor of a concerned party. To mitigate this informational problem, the European Competition Authority has recently decided to create its own team of economic experts. ${ }^{2}$ Other examples of delegated expertise when some experts are biased are, among others, stock recommendations by financial analysts, ${ }^{3}$ the refereeing process of academic journals or the evaluation of the impacts of climate change by scientific experts or environmental agencies.

This paper studies how those conflicts of interests affect the interactions between the experts and the decision maker when the formers also care about their reputation on the market for experts. We analyze the effect of the expertise procedure on the efficiency of information transmission. We

\footnotetext{
${ }^{1}$ Statement of Linda A. Suydam, Senior Associate Commissioner, Food and Drug Administration - Department of Health and Human Services, before the House Committee on Government Reform on June 14, 2000.

${ }^{2}$ See Roller, Stennek and Verboven (2001), Kuhn (2002) and Rey (2003) for more details about European Competition Authority decision.

${ }^{3}$ Bruce (2002) analyzes what causes analysts to have such a significant bias in their recommendations. See also Morgan and Stocken (2003) for a theoretical study of this point.
} 
focus on the nature of the informational linkage with the market for experts, when the information provided by experts is verifiable. In other words, experts provide evidence as opposed to opinions.

We develop a framework where a principal hires, at some fixed wage, an outside expert, who cares about his reputation, the social surplus, and also his own interests. For instance, he may have common interests with a concerned party, the competitors or the consumers. Experts may gather a private signal about the principal's surplus, which is hard information. Thus the expert cannot manipulate his signal, but he may conceal it. Within this framework, the principal will always follow an expert's advice when it is informative. Reputation effects arise because the expert's ability to generate an informative signal is unknown and evidence of information gathering signals this ability to the market. Such evidence may directly stems from disclosure of the expert's report's informativeness, or indirectly from its effect on the decision.

We first analyze the case in which the market perfectly observes the expert's reports, identity and bias, referred to as full transparency. If the expert's bias is not too high, he will always report the truth. But an expert may have incentives to misreport when his bias is high. Indeed, when misreporting moves the decision toward his preferred one, the expert may conceal his information. Less information is then transmitted when the bias moves toward a stronger preference for the "status quo." We also show that misreporting is, to some extent, self-enforcing: the more the market anticipates some misreporting, the higher the incentives to misreport. ${ }^{4}$ The reason is that the reputation loss from not reporting decreases when the informed expert misreports more.

We then extend the model in several dimensions, corresponding to various aspects of expertise procedures.

First we find that it is not optimal to hide the identity of the expert nor the nature of his report. The simple intuition is that limiting the public access to information would make misreporting less costly for the expert in terms of reputation.

We then generalize the analysis in introducing uncertainty on the experts' bias. Surprisingly, it is not always the case that transparency on the bias leads to more revelation of information. The reason lies in the self-enforcing aspect of misreporting mentioned above. When the bias is uncertain, the reputation loss for no report is independent of the bias, and for some intermediate range of bias larger than if the bias was revealed leading to less misreporting. The overall effect is ambiguous and depends on the distribution of the bias.

\footnotetext{
${ }^{4}$ This creates the possibility of multiple equilibria even though we characterize necessary and sufficient conditions under which a unique equilibrium exists.
} 
Finally, we examine multiple experts procedure, by allowing the principal to rely on two experts, with different bias and quality of expertise, and to choose the timing of expertise (simultaneous or sequential). In this set-up we show that under full transparency, the presence of a second expert reduces the incentive of an expert to misreport. This is due to the fact that the probability that an uninformed report moves the decision is reduced. In terms of information revealed, a procedure with simultaneous expertise leads to more information revealed than one with sequential expertise, although if the costs of expertise are high, the optimal timing may be the sequential one.

In a contrasting way, when experts' reports are secret, hiring a second expert reduces the first expert's incentives to report his information. The intuition behind this result is that the signal transmitted to the market on the expert is garbled if a second expert is hired, because the market observer doesn't know which expert generated a decisive information. Caeteris paribus, hiring a second expert is less attractive if the reports are secret compared to the case in which they are public. In this case, the sequential timing dominates both in terms of information revelation and costs of consultation.

The paper is organized as follows. Section 3 describes the base model which is solved under full transparency in the following section. Section 5 analyzes the disclosure of information to the market, while section 6 derives the equilibrium under imperfect information on the bias. The procedure with multiple experts is discussed in section 7 .

\section{Related literature}

This paper is at the frontier between two streams of literature on decision making with strategic disclosure of information by interested (biased) parties: the literature focusing on verifiable information initiated by Grossman (1981) and Milgrom (1981), and the literature on the reputation of experts initiated by Sobel (1985) or Ottaviani and Sorensen (2001). It shows how reputation effects the extent of "sanitazition" that the expert It points to an intricate relationship between the bias of the expert and the

. In this persuasion game framework, Milgrom and Roberts (1986) show that strategic decision makers are skeptical i.e. they consider any missing information as unfavorable. Moreover, there is 
full revelation of the information in the unique equilibrium. Shin (1994) shows that full revelation does not survive to the introduction of uncertainty about the expert's information. Dur and Swank (2005) point out that it might be optimal to hire experts with bias different from the decision maker in order to improve incentives to acquire information. We show in this paper that those results are robust to the introduction of reputation on the expert's side and to the limitation of the information disclosed to the market for experts. Wolinsky (2003) discusses information transmission without reputation effects when the experts' bias are uncertain and when information is verifiable in the sense of Shin (1994). A biased expert reports less favorable information than when her bias is known while we show that in all pure strategies equilibria, the most biased experts lie less with uncertain bias.

The nearest antecedent to our paper is Dewatripont and Tirole (1999) who analyze the advantages of competing advocacies in an incomplete contract framework with verifiable information. They show that, in selecting two competing agents each collecting one signal rather than one gathering two signals, the principal may improve the quality of decision-making by raising incentives. Our paper deviates from theirs in that we don't model the expert incentives to generate the information but only to divulge it. Moreover we assume that all experts gather the same information, which fits for instance the case of medical expertise, or security expertise. Second, we analyze the efficiency of information transmission between the experts and the principal under different informational features while Dewatripont and Tirole (1999) concentrate on the case of public reports. ${ }^{5}$

Information transmission between an interested expert and a decision maker also has been studied by Kofman and Lawarree (1993) and Gromb and Martimort (2007) among others in a mechanism design framework where information is contractible. Moreover, several authors have analyzed the incentives of biased experts to misreport in cheap talk models. ${ }^{6}$ The main difference with our paper is that information is hard in our model even though it can be concealed. In our motivating examples, the experts have to provide formal arguments when they send a report to the decision maker and the decision maker cannot directly contract on the information provided by the expert; that is the reason why we adopt this model specification.

Crawford and Sobel (1982) show that full reporting of information is impossible in cheap talk models when an expert is biased. Krishna and Morgan (2001) and Li (2009) extend this result

\footnotetext{
${ }^{5}$ Beniers and Swank (2004) analyze the optimal composition of committees in a similar framework.

${ }^{6}$ This literature has been initiated by Crawford and Sobel (1982).
} 
and show that consulting two experts is better than consulting just one when experts are biased in opposite directions. In this paper, we show that their result establishing that hiring two experts is more efficient than hiring only one is true exclusively in case reports are public. Specifically, this result cannot be generalized to the case of secret reports.

Sobel (1985) and Morris (2001) introduce reputation concerns in repeated cheap talk games. However, in their model, reputation concerns are about the bias of the expert while in our model it is about the quality of expertise. This reverses the effects of reputation. In our model reputation induces the expert to report the truth more often. We also share Ottaviani and Sorensen's (2001, $2006 \mathrm{a}$ and $2006 \mathrm{~b}$ ) result that reputation concerns does not give in general the right incentives to truthfully report information. However, we mitigate this result by accounting for some altruism in the experts objective. In our model, the expert always report truthfully for low values of the bias. The timing of consultation when experts care about their reputation is also analyzed by Ottaviani and Sorensen (2001). They show that reputation may lead to herding problems when experts are consulted sequentially. In our model, this herding effect does not exist because hard information prevents experts to lie in order to appear correct and the optimal timing depends on the informational structure. ${ }^{7}$

\section{The model}

A decision authority, hereafter the principal or she, has to make a decision $d$ that can take two values, $d=0$ or $d=1$. The principal is risk neutral. Her objective is to maximize the social surplus, ${ }^{8}$ d.v, where $v$ is unknown, with a finite and positive mean. Thus in the absence of any information, the principal would choose $d=1$, referred to as the status quo.

To reach an efficient decision, the principal hires at some fixed wage an expert. The expert gathers an informative signal with some probability $p$, or no signal with probability $1-p$. The expert information is thus $s \in\{\emptyset\} \cup \mathbb{R}$ where $s=\emptyset$ corresponds to no signal. When $s \in \mathbb{R}$, the signal is informative and we denote $\theta=E(v \mid s)$. In what follows we identify an informative signal with the mean value of the status quo decision: $s=\theta$. We assume that $\theta$ is a parameter continuously distributed on the real line, with a cumulative distribution function $F$ and a density function $f($.$) .$

\footnotetext{
${ }^{7}$ See also Prendergast (1993).

${ }^{8}$ In what follows we assume that the principal doesn't internalize the welfare of the expert. We develop in appendix a model where the principal accounts for the expert's utility that yields the same objective functions.
} 
Upon observing or not the signal, the expert makes a report $r$ to the principal. The expert cannot report that he has gathered an informative signal if it is not the case, nor report another informative signal than the one he has gathered. In other words the informative signal is hard information and can be transmitted to the authority. An interpretation for hard information is that the principal requires a formal proof in order to validate the expert's report; another is that falsification of the information is prohibited by law and lying is therefore too costly for the expert. However, an expert receiving an informative signal can hide this and choose not to report the value of $\theta$ by claiming the signal was uninformative. ${ }^{6}$ Such a strategy of removing bad news form reports is refered to a as a "sanitization" strategy by Shin (1994). Thus the expert reports $r \in\{\emptyset, s\}$. It follows that when the expert transmits an informative signal $r \neq \emptyset$, the optimal decision in unambiguously to choose the decision that maximizes $d . r$ We say in this case that the expert's report is decisive.

As an example, consider a pharmaceutical company that has conducted a first clinical trial that led to the posterior $E(v)$ for the social value of a new treatment. The company obtains positive profit if the treatment is introduced. An expert is asked to run a new trial, who may report conclusive results or claim that the clinical trial failed to be conclusive. The expert is dependent of the pharmaceutical company for some of his income and thus interested in the profit of this company.

Another example would be an econometrician running a SSNIP test in a merger case, but who is active in consulting and has some connexion with the industry. The econometrician may then choose to report significant estimates, or to report only a poor econompetrci study with no significant coefficients.

The expert cares about social welfare but he is biased and receives a benefit $\beta$ when the decision $d=1$ is reached. We assume that the bias is known and will discuss the effect of imperfect information (transparency on the bias). The expert also cares about its reputation on the market for experts.

Prior to the consultation, the expert's level of expertise is unknown to all the parties. Expertise can be high, in which case the expert gathers an informative signal with probability $\bar{p}$, or low, in which case the probability is $\underline{p}$. Let $\pi$ denote the prior probability that an expert has high expertise. The prior probability that the expert be informed is $p^{e}=\pi \bar{p}+(1-\pi) \underline{p}$. His future prospects depend

\footnotetext{
${ }^{6}$ This framework is also used in Shin? Laffont and Tirole (1991) and Dewatripont and Tirole (1999).
} 
on the market perception of his ability. Following Ottaviani and Sorensen (2001, 2006a, 2006b), we assume that reputation is captured by the probability that the market assigns to the expert being of high expertise, and that his expected utility is linear in this probability. In particular the expert obtains a premium $R$, representing the increase in the value of his future revenues, when being perceived of high expertise with probability 1 . The expert is risk neutral so that, letting $I$ denote the ex-post information of the market on the level of expertise, the objective of the expert is to maximize the expectation of the following utility function:

$$
U=\beta d+\theta d+R \operatorname{Pr}(p=\bar{p} \mid I)
$$

where the first term corresponds to the expert's bias, the second to the weight he puts on the social surplus, the third to his willingness to maintain his reputation. Notice that the utility doesn't incorporate monetary transfer. While we do not rule out monetary transfers, we assume that there cannot be made contingent on the report or on the final decision. Thus they reduce to a fixed wage determined ex-ante.

Our basic model assumes that all the relevant information is divulged. Concretely this means that the principal discloses publicly the identity of the expert appointed, and also whether the information provided by the expert is decisive. The game is then the following:

i) in a first stage, nature draws the value of $\theta$ and the expert observes the signal $s \in\{\emptyset, \theta\}$ and subsequently makes a report $r \in\{\emptyset, \theta\}$;

ii) the principal chooses $d$;

iii) finally, the market observes whether the report is decisive, update the beliefs and payoffs are realized.

\section{Strategies}

A (pure) strategy for an expert is thus a function mapping the expert's private information $s$ about $\theta$ into a report. It is fully characterized by the set $\Theta$ of informative signals for which an informed expert decides to report no information. For other signals, $s$ is truthfully reported. For conciseness reasons, we focus attention to equilibria where the informed expert reports truthfully when he is indifferent between reporting or not, provided that such an equilibrium exists. This is innocuous for the case in which the report is public, but it rules out some pathological equilibria in the case of secret reports. 
The strategy of the authority is a mapping from reports into probabilities of making decision $d=1$. As information is hard and the principal cannot commit ex-ante to the strategy, when the expert's report is decisive, the principal follows the advice and the corresponding decision is $d=1$ (resp. $d=0$ ) if the expert reports the signal $\theta>0$, (resp. $\theta<0)$. However, when the expert reports an uninformative signal $\emptyset$, then the principal decides according to his posterior beliefs. We denote by $q$ the probability that the principal chooses the status quo $d=1$ when there is no decisive report.

\section{Equilibrium under full transparency}

When reports are public, notice that the report is sufficient for the market to update its beliefs. In particular knowledge of the decision adds no information. Moreover the value $\theta$ for a decisive report is also irrelevant for determining the market posterior belief, as this doesn't add any useful information on the type of the expert to the knowledge that he was informed. In other words, the market's posterior beliefs about the type of the expert is the same for all decisive reports. We denote by $\Delta$ the reputation gain obtained by an expert when he is decisive. It is proportional to the difference between the posterior probability that the expert be of high type when the report is decisive, and the posterior probability when the expert reports no information. Formally, since a report $r=\emptyset$ occurs if $s=\emptyset$ or $s \in \Theta$, the equilibrium value of $\Delta$ is equal to:

$$
\begin{aligned}
\Delta & =R\left[\operatorname{Pr}\left(p=\bar{p} \mid s \in \mathbb{R} \backslash \Theta_{N R}\right)-\operatorname{Pr}\left(p=\bar{p} \mid s \in\{\emptyset\} \cup \Theta_{N R}\right)\right]>0 \\
& =R(1-\pi) \pi \frac{\bar{p}-\underline{p}}{p^{e}\left(1-p^{e}+p^{e} \operatorname{Pr}(\Theta)\right)} .
\end{aligned}
$$

Thus in equilibrium $\Delta$ is positive and decreasing with the probability that $\theta$ belongs to the set $\Theta_{N R}$.

While announcing the true $\theta$ always induces the "correct" decision $d=0$ or 1 , concealing the information received may generate an inefficient decision. Comparing the expert's utility from reporting to his utility from not reporting, we see that the expert's gain from telling the truth is

$$
\left\{\begin{array}{c}
(\beta+\theta)(1-q)+\Delta \text { when } \theta \geq 0 \\
-q(\beta+\theta)+\Delta \text { when } \theta<0
\end{array}\right.
$$

where, recall, $q$ is the probability that the decision is $d=1$ when there is no decisive report. In equilibrium, the expert hides information whenever this gain is negative while $q$ maximizes $q E(\theta \mid s \in\{\emptyset\} \cup \Theta)$. 
The way in which the principal acquires information from the experts' reports is twofold. First, when reporting allows the expert to reach his favorite decision or when misreporting may cause too much social damage, the expert truthfully reports the information. This enables the principal to make the right decision. Second, when reporting induces the principal to choose the expert's least favored decision, the expert may withhold the information. Even though the authority cannot distinguish when the expert misreports or when he has not gathered any information, she gives more importance to the states of the nature belonging to $\Theta$ when making her decision. Decision making is therefore always more efficient than without any experts. The efficiency gain depends on the extent of misreporting.

It is immediate that if the expert is biased against the status quo $(\beta \leq 0)$, there is an equilibrium where the expert always announces the true signal and the correct decision is taken. The reason is that the expert cannot induce a more favorable decision by misreporting as this strengthens the prior idea of the principal. In such an equilibrium, the status quo is chosen if there is no report and there is no incentive to hide the information. More generally we can easily see that

Lemma 1 There exists an equilibrium where the expert fully reveals his information if and only if $\beta \leq \Delta_{0}=R(1-\pi) \pi \frac{\bar{p}-\underline{p}}{p^{e}\left(1-p^{e}\right)}$.

Proof. Suppose the market anticipates that $\Theta=\varnothing$. Then $q=1$ and $\Delta=\Delta(\varnothing)$. The expert reveals $\theta \geq 0$ since $\Delta$ is positive and reveals all $\theta<0$ iff $\beta \leq \Delta_{0}$.

When the private benefits received by an expert if his preferred decision is reached are low, then the reputation effect induces full revelation of the expert's information. Intuitively, as in such cases the gains from moving a decision are low, it is in the expert's interest to enhance his reputation by reporting truthfully.

\subsection{Equilibrium with misreporting}

The interesting case arises when the expert is strongly biased in favour of the status quo, so that the expert may be tempted to hide a negative information. So let us now focus on the case in which $\beta>\Delta_{0}$.

Then an expert informed that the socially optimal decision corresponds to his preferred one always reports it to the principal. Indeed, making an uninformative report reduces the reputation 
term and doesn't bring any gains in terms of decision. This is the case if $s=\theta \geq 0$. This is also the case when $\theta \leq-\beta$ since then the final decision $d=0$ corresponds to his preferred decision. This defines a lower bound for the values of efficiency gains for which an expert lies: $\Theta \subset(-\beta, 0)$. But the expert may be tempted to induce $d=1$ by not reporting a negative signal if the social cost of the status quo is small.

On the range of values between $-\beta$ and 0 , the expert doesn't report truthfully when the gain from inducing the status quo outweighs the cost in terms of reputation. Since reporting induces $d=0$, the gain in (2) is positive and the expert truthfully reports a negative signal if

$$
q(\beta+\theta) \leq \Delta
$$

There are two straightforward consequences for equilibrium behavior.

First, in any equilibrium the status quo is chosen with positive probability if the report is not decisive, $q>0$. The reason is that $q=0$ would induce the expert to always reveal his signal, which is not possible in equilibrium by definition of the status quo.

Second, it must be the case that the expert misreports for values $\theta$ in an interval $\Theta=\left(\theta^{p}, 0\right)$. We then have $\Delta=\Delta^{p}\left(\theta^{p}\right)$ :

$$
\Delta^{p}\left(\theta^{p}\right)=\frac{\Delta_{0}}{1+\frac{p^{e}}{1-p^{e}}\left(F(0)-F\left(\theta^{p}\right)\right)} .
$$

This function measures the net reputation gain for an unbiased expert of revealing that the value is $\theta^{p}<0$, when the status quo is a decision $d=1$ and beliefs are that $\left(\theta^{p}, 0\right)$ is not revealed. Notice that $\Delta(\varnothing)$ is the value of the reputation gain when the fact that the expert receives the information or not is public.

A biased expert will trade-off the reputation gain with the cost of inducing the least preferred decision $d=0$. We then denote by $v\left(\theta^{p}\right)=E\left(\theta \mid s \in\{\emptyset\} \times\left(\theta^{p}, 0\right)\right)$ the authority's expected value of the decision $d=1$ when receiving an uninformative report $r=\emptyset$. Equilibrium conditions are then condition 3 along with the following:

i) Either $v\left(\theta^{p}\right) \geq 0, q=1$, and

$$
\beta+\theta^{p}=\Delta^{p}\left(\theta^{p}\right)
$$

ii) or $v\left(\theta^{p}\right)=0$ and

$$
0<q=\frac{\Delta^{p}\left(\theta^{p}\right)}{\theta^{p}+\beta}<1 .
$$


We refer to the latter equilibrium as a mixed strategy equilibrium.

There is a non-trivial relationship between the expert's preference (the bias $\beta$ ) and the reputation effect which may result in two interesting phenomena: mixed strategy and multiple equilibria.

\section{Random choice by the principal}

When the bias $\beta$ is large and/or the reputation effect is small, the expert would not report a large set of negative values if it were inducing the principal to choose the status quo. Then no report would be interpreted as a bad new for the status quo. In this the expert's incentives to report are fostered by reducing the predictability of the decision of the principal making the status quo less likely. This is only possible if $v\left(\theta^{p}\right)=0$. Let us remark that $v\left(\theta^{p}\right)$ increases with $\theta^{p}$ and $v(0)=E(\theta)$. Thus there is at most one mixed strategy equilibrium. But it may coexist with pure strategy equilibria.

When $v(-\infty)<0$, we define $\theta_{0}$ as the (unique) solution of

$$
v\left(\theta_{0}\right)=0
$$

By convention, set $\theta_{0}=-\infty$ when $v(-\infty) \geq 0$. Then it is immediate that $\theta^{p} \geq \theta_{0}$ with equality in the case of a mixed strategy equilibrium. In particular there is no mixed strategy if $v$ is non-negative everywhere, which occurs if $p^{e}$ is small.

\section{Multiple equilibria}

A key point concerning reputation and reports, is that the more the expert misreports, the lower the cost in terms of reputation associated with misreporting. This is because it becomes relatively less likely that a non-decisive expert didn't receive any information. Indeed, the fact that the expert misreports for some values of the signal reduces the opportunity cost of not reporting as it becomes relatively less likely that a non-reporting expert didn't receive any information. This creates an interaction between the expert's bias and the reputation effects. More precisely, when the market anticipates that the set of informative signals for which an informed expert decides to misreport is large, there is little updating of expertise from misreporting and hence, the reputation effects are small which reduces the expert's incentives to report the true signal. In contrast, when the market anticipates that the expert reports the truth more often, the loss of reputation faced by an expert who misreports is larger and the expert's incentives to report the true signal increase.

Thus misreporting is to some extent self-reinforcing: the more the market anticipates some misreporting, the higher the incentives to misreport. This creates the possibility of multiple equi- 


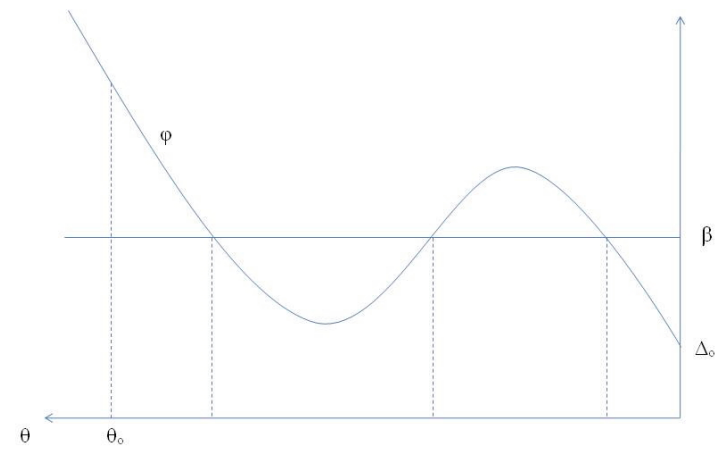

libria. Notice that the structure of equilibria is governed by the shape of the function $\Delta^{p}(\theta)-\theta$. A pure strategy equilibrium is such that $\beta=\Delta^{p}\left(\theta^{p}\right)-\theta^{p}$ and several equilibria may therefore emerge depending on the variations of this function. An example of multiple equilibria is presented below.

Uniqueness of the equilibrium results if $F$ satisfies a standard monotone hazard rate condition:

Assumption A For $\theta<0: \frac{\partial}{\partial \theta}\left[\frac{f(\theta)}{F(0)-F(\theta)}\right] \geq 0$.

We show in appendix that this condition implies that the function $(\beta+\theta) / \Delta^{p}(\theta)$ is quasiconcave.

The following proposition then follows from the previous equilibrium conditions.

Proposition 1 When $\beta>\Delta_{0}$, there exists an equilibrium characterized by misreporting for $\theta \in$ $\left(\theta^{p}, 0\right)$. Under assumption $A$, the equilibrium is unique ${ }^{9}$ and:

i) $q=1$ and $\phi\left(\theta^{p}\right)=\beta$ if $\Delta^{p}\left(\theta_{0}\right)-\theta_{0} \geq \beta$;

ii) $q<1$ and $\theta^{p}=\theta_{0}$ if $\Delta^{p}\left(\theta_{0}\right)-\theta_{0}<\beta$.

Proof. Notice that for $\theta \leq-\beta: \Delta^{p}(\theta)-\theta>\beta>\phi(0)$. Thus either $\theta_{0}>-\beta$ and $\Delta^{p}\left(\theta_{0}\right)-\theta_{0}<$ $\beta$ or there exists $\theta^{p} \geq \theta_{0}$ solution of $\phi\left(\theta^{p}\right)=\beta$. In the former case $\theta_{0}$ and $q=\frac{\phi\left(\theta_{0}\right)+\theta_{0}}{\beta+\theta_{0}}$ is an equilibrium, while in the latter case $\theta^{p}$ and $q=1$ is an equilibrium.

\footnotetext{
${ }^{9}$ Another sufficient condition for uniqueness is that the information is dispersed enough so that $\Delta_{0} f(\theta)<1$ on $(-\beta, 0)$.
} 
We show in appendix that $(\theta+\beta) / \Delta^{p}(\theta)-1$ is strictly quasi-concave under assumption $\mathrm{A}$ with negative value at $-\beta$ and positive value at 0 . Thus there exists a unique solution $\theta \in(-\beta, 0)$ to $(\theta+\beta) / \Delta^{p}(\theta)-1=0$.

The equilibria are depicted in Figure 1 for the case in which $\Delta$ has a slope smaller than 1.

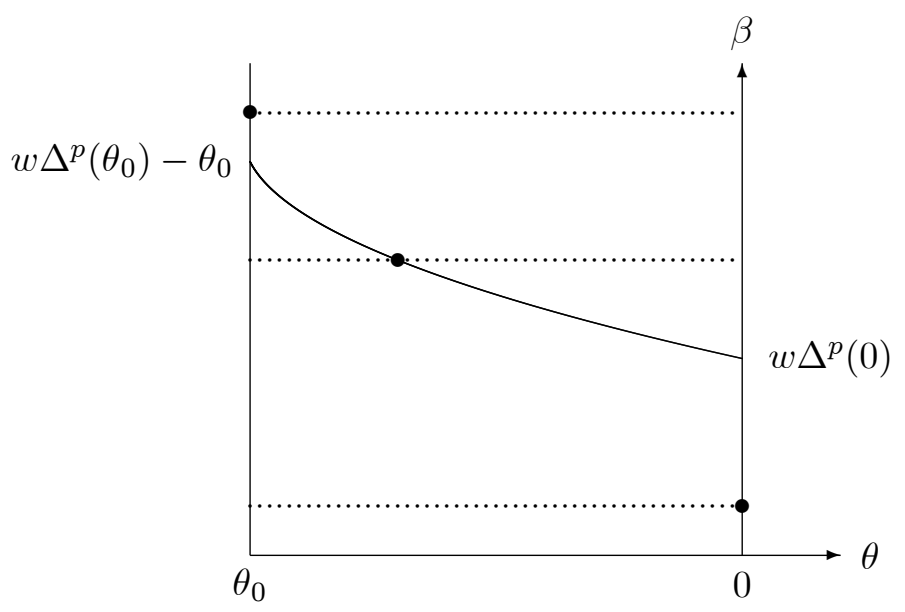

Fig. 1: Equilibria with $\Delta^{p \prime}(\theta) \leq 1$.

Remark 1 Assumption $A$ don't warrant uniqueness of the equilibrium if $\beta<\Delta_{0}$, which would require that $\Delta^{p}-\theta$ be decreasing and not only convex. When $\beta<\Delta_{0}$, there may exist two equilibria with misreporting along with the equilibrium with full reporting. In all that follows, we focus on the most informative equilibrium, namely the one with full reporting.

\subsection{The value of expertise}

In order to analyze the effects of the conflict of interests on the different parties, we now examine how the information revealed by the expert varies with his characteristics. Without expertise the principal's welfare would be $E(\theta)$. The expert then reverts the decision with probability $p^{e}$ if $\theta<\theta^{p}$, inducing a welfare gain $-\theta>0$. For $\theta>\theta^{p}$ there is no welfare gain for the principal since either $d=1$ or the principal is indifferent between $d=1$ and $d=0$. Thus the principal's welfare gain from expertise, gross of the transfer to the expert if there is one, writes as

$$
V_{P}=p^{e}\left(-\int_{+\infty}^{\theta^{p}} \theta f(\theta) d \theta\right)
$$


This term represents the value of the expertise for the principal when she doesn't internalize the welfare of the expert. With this interpretation we don't face the issue of evaluating the bias. Another interpretation could be that the principal internalizes the welfare of the expert with a proper normalization of utilities and transfers. This is developed more extensively in our 2010 working paper.

In any case the welfare gain is that for reported negative values of $\theta$ the decision is reversed. It is immediate that the principal's welfare increases with $\theta^{p}$ and $p^{e}$. In particular in the case of multiple equilibria, wethe welfare gain $V_{P}$ is the highest for the largest value of $\theta^{p}$, thus when the extent of misreporting is minimal. ${ }^{10}$

Assuming a unique pure strategy equilibrium, we then have

Corollary 1 Assume $A$ and $\Delta^{p}\left(\theta_{0}\right)-\theta^{p} \geq \beta>\Delta_{0}$. Then $\theta^{p}$ decreases with $\beta$ and increases with $R$.

Proof. Increasing $R$ shifts $\Delta$ upward which shifts the curve $\theta^{p}$ to the right in the $\left(\beta, \theta^{p}\right)$ plan, similarly for a reduction in the bias. The effect is neutral on $\theta^{p}$ if $q<1$ since $\theta_{0}$ is independent of $R$ and $\beta$.

Thus without surprise, the principal prefers an expert with little bias and high reputation concerns.

Notice also that if we fix the average quality $p^{e}$, the value of reputation evolves with the underlying characteristics of the expert as $\Delta_{0}$, the reputation effect under truthtelling. This latter depends on the quality differential between the two types of experst and on the information conveyed by the report of a signal on the expertise:

Corollary 2 Assume $A$ and $\Delta^{p}\left(\theta_{0}\right)-\theta_{0} \geq \beta>\Delta_{0}$. For a fixed average quality $p^{e}$, increasing the quality differential $\bar{p}-\underline{p}$ raises $\theta^{p}$; the same holds for an increase of $\pi(1-\pi)$.

Proof. The function $\Delta$ increases and thus $\theta^{p}$ decreases when $\Delta_{0}=(1-\pi) \pi \frac{\bar{p}-\underline{p}}{p^{e}\left(1-p^{e}\right)}$ increases.

Notice that the variance of $p$ is $(1-\pi) \pi(\bar{p}-\underline{p})^{2}$. Thus the interpretation is that due to stronger reputation effects, the extent of revelation of the expert's information increases when the dispersion

\footnotetext{
${ }^{10}$ We should point that there is no Pareto ranking of equilibria in this case. The principal prefers the most informative equilibrium, but the expert prefers the lowest equilibrium value of $\theta^{p}$.
} 
of expertise increases. To this extent, larger uncertainty on the quality of the expert improves the quality of expertise.

The last question is whether the principal prefers better experts. For this notice that for a fixed quality differential and probability $\pi$, increasing $p^{e}$ has two effects. First it raises the probability that the expert learn the value $\theta$. Second, it affects the inference by reducing the likelihood that a non-decisive report is due to bad luck instead of strategic manipulation of information.

Corollary 3 Assume $A$ and $\Delta^{p}\left(\theta_{0}\right)-\theta_{0} \geq \beta>\Delta_{0}$. Then $V_{P}$ is increasing with the average quality of the expert if $p^{e}>\frac{1}{2(1-F(0))}$ (holding fixed the quality differential and the variance).

Proof. Indeed

$$
\begin{gathered}
\Delta^{p}(\theta)=\frac{R(1-\pi) \pi(\bar{p}-\underline{p})}{p^{e}\left(1-p^{e}+p^{e}(F(0)-F(\theta))\right)}-\theta ; \\
\operatorname{sign}\left[\frac{\partial \Delta^{p}(\theta)}{\partial p^{e}}\right]=\operatorname{sign}\left[2 p^{e}(1+F(\theta)-F(0))-1\right]
\end{gathered}
$$

It is positive if $p^{e}>1 / 2(1-F(0))$ implying the $\Delta$ increases and thus $\theta^{p}$ increase with $p^{e}$.

However $\Delta$ decreases with $p^{e}$ at least for $p^{e} \leq 1 / 2$. In this case $\theta^{p}$ decreases which $p^{e}$ so that the overall effect is ambiguous since increasing $p^{e}$ raises the probability that the expert obtains the information but reduce the likelyhood that he reveals it. Indeed

$$
\frac{\partial V_{P}}{\partial p^{e}}=-\int_{+\infty}^{\theta^{p}} \theta f(\theta) d \theta-\theta^{p} f\left(\theta^{p}\right) p^{e} \frac{\partial \theta^{p}}{\partial p^{e}},
$$

which may be positive or negative. Notice that $\Delta^{p}\left(\theta^{p}\right)$ depends only on the distribution of $\theta$ between $\left(\theta^{p}, 0\right)$, i.e on the restriction of $f$ on $\left(\theta^{p}, 0\right)$. Thus for any distribution such that

$2 p^{e}\left(1+F\left(\theta^{p}\right)-F(0)\right)-1<0$ but $\frac{\partial V_{P}}{\partial p^{e}}>0$ there exists a distribution with the same $\theta^{p}$ and different density below $\theta^{p}$ and above zero sucht that $\frac{\partial V_{P}}{\partial p^{e}}<0$.

\section{$5 \quad$ Transparency on the bias}

As mentioned in the introduction, most expertise procedures require the expert to report potential conflicts of interest. To address this issue we relax the previous assumptions by assuming that the bias is private information of the expert. For this purpose, we assume that the prior information of both the principal and the market on the expert's bias is represented by a smooth distribution whose support is an interval $[\beta, \bar{\beta}]$ where $\bar{\beta}>0$. We also assume that the reputation gain $R$ of the 
expert is known, and not related to the value of $\beta$. This assumption rules out issues of strategic manipulation of beliefs on $\beta$ as analyzed in the work of Sobel (1985) or Morris (2001).

The equilibrium behavior of the expert is the same as before, but now the level $\theta^{p}$, at which the expert is indifferent between informing the principal or not, depends on the bias $\beta$, while the decision of the principal and the reputation effects are based on mean values.

We denote as above $\Delta$ the equilibrium reputation gain from telling the truth and $q$ the probability that the principal faced with no decisive report chooses the status quo. The expert of type $\beta$ truthfully reports a negative $\theta$ if $q(\theta+\beta) \leq \Delta$. Thus there exists a threshold $z=\Delta / q$, such that the expert's report is not decisive if

$$
z-\beta<\theta<0
$$

Notice that the expert may hide information only if $z$ is smaller than $\bar{\beta}$, and that the lower the bias $\beta$ the more information is revealed. We can then define the reputation gain as:

$$
\begin{aligned}
\Delta^{u}(z) & =R[\operatorname{Pr}(\bar{p} \mid r \neq \emptyset)-\operatorname{Pr}(\bar{p} \mid r=\emptyset)] \\
& =R\left[\frac{\pi \bar{p}}{p^{e}}-\frac{\pi[1-\bar{p}]+\pi \bar{p} E_{\beta}[\max \{F(0)-F(z-\beta), 0\}]}{1-p^{e}+p^{e} E_{\beta}[\max \{F(0)-F(z-\beta), 0\}]}\right]
\end{aligned}
$$

which can be written as

$$
\Delta^{u}(z)=\frac{\Delta_{0}}{1+\frac{p^{e}}{1-p^{e}} E_{\beta}[\max \{F(0)-F(z-\beta) ; 0\}]} .
$$

An equilibrium can then be characterized by a pair $\left(q^{u}, z^{u}\right)$ such that the condition

$$
\Delta^{u}\left(z^{u}\right)=q^{u} z^{u}
$$

holds and $q^{u}$ is optimal for the principal given that the expert hides the information $\theta \in\left(z^{u}-\beta, 0\right)$. The optimal choice of $q^{u}$ depends on the expected value of $\theta$ when no information is revealed by the expert:

$$
E\left[\theta \mid s \in\{\emptyset\} \cup\left(z^{u}-\beta, 0\right)\right]
$$

where the expectation accounts for distribution of both $\theta$ and $\beta$. This is increasing with $z^{u}$ and following the same reasoning as in the previous section, we can define the critical value $z_{0}$ (possibly infinite) as the level such that $E\left[\theta \mid s \in\{\emptyset\} \cup\left(z^{u}-\beta, 0\right)\right] \leq 0$ if and only if $z^{u} \leq z_{0}$. An equilibrium with $q^{u}<1$ is only possible if $z_{0}$ is finite and $z^{u}=z_{0}$.

With these notations the equilibrium conditions for an equilibrium write as follows 
i) Either $q^{u}=1$ and $z^{u} \geq z_{0}$ is the solution of the equation $\Delta^{u}\left(z^{u}\right)=z^{u}$;

ii) or $z^{u}=z_{0}>\Delta^{u}\left(z_{0}\right)$ and $0<q^{u}=\frac{\Delta^{u}\left(z_{0}\right)}{z_{0}} \leq 1$.

The analysis is thus the same as before but adjusting for the new function $\Delta^{u}(z)$ and threshold $z_{0}$, which incorporate the uncertainty about the bias in the reputation gain and the principal decision process.

Proposition 2 Assume that $\beta$ is uncertain, then there exists an equilibrium and:

1. There is an equilibrium where the expert always reports truthfully his information if and only if $\bar{\beta} \leq \Delta_{0}$.

2. When $\bar{\beta}>\Delta_{0}>z_{0}$, the expert misreports for $\theta \in\left(z^{u}-\beta, 0\right)$ where $z_{0} \leq z^{u}<\Delta_{0}$.

3. When $\bar{\beta}>z_{0} \geq \Delta_{0}$, the expert misreports for $\theta \in\left(z_{0}-\beta, 0\right)$

Proof. Follows from above. Notice that $\Delta^{u}(z)=\Delta_{0}$ if and only if $z \geq \bar{\beta}$. Moreover $z_{0}<$ $\bar{\beta}$. When $\bar{\beta}>\Delta_{0}$, we must thus have $z^{u}<\bar{\beta}$. The function $\Delta^{u}(z)-z$ is negative at $z=\bar{\beta}$, therefore if $\Delta^{u}\left(z_{0}\right)-z_{0}>0$ there exist a solution $z^{u}$ to $\Delta^{u}\left(z^{u}\right)-z^{u}=0$. Since $\Delta^{u}(z)<\Delta_{0}$, we have $z_{u}=\Delta^{u}(z)<\Delta_{0}$ if $z_{0}<z_{u}$ which is possible only if $z_{0}<\Delta_{0}$. Moreover if $z_{0}>\Delta_{0}$ then $z_{0}$ is the unique solution to equilibrium conditions.

This result has some interesting policy implications. First truth telling when $\beta$ is unknown can be an equilibrium only if it is one for all possible values of $\beta$. To this respect lack of transparency on the bias reduces information revelation on the correct decision.

Moreover when $\bar{\beta}>\Delta_{0}$, the fact that $z^{u}<\bar{\beta}$ implies that $\Delta_{0}>\Delta^{u}$. Hence the fact some expert don't report truthfully reduces the reputation for all experts. Hence an expert may decide to hide some information when the bias is uncertain although he would report all the information if his bias were plublicly knonw. This occurs for an expert with a bias in the range

$$
z^{u}<\beta \leq \Delta_{0}
$$

To further highlight the effect of transparency, we will now compare for $\beta>\Delta_{0}$, the extent of misreporting by comparing the value $\theta^{p}(\beta)$ when $\beta$ is publicly known with the value $z^{u}-\beta$ obtained when $\beta$ is private information. There is more information generated by the expertise with 
transparency on the bias for values of $\beta$ such that if $z^{u}-\beta<\theta^{p}(\beta)$. Since $z^{u}$ is constant, we study the variations of $\theta^{p}(\beta)+\beta$.

To perform the comparison we assume that assumption $\mathrm{A}$ holds so that $\theta^{p}(\beta)$ is uniquely defined. Then on the range where $\theta^{p}(\beta)<\theta_{0}$ we have $\theta^{p}(\beta)+\beta=\Delta^{p}\left(\theta^{p}(\beta)\right)$ which is decreasing with $\beta$ since $\theta^{p}(\beta)$ decreases and $\Delta^{p}(\theta)$ increases. When $\theta_{0}$ is finite the minimum is reached $\Delta^{p}\left(\theta_{0}\right)$. When $\theta_{0}$ is infinite, then $\theta^{p}(\beta)$ tends to $-\infty$ when $\beta$ converges to infinity, hence $\theta^{p}(\beta)+\beta$ converges to $\Delta^{p}(-\infty)=\lim _{\theta \rightarrow-\infty} \Delta^{p}(\theta)$.

We thus concludes that $\theta^{p}(\beta)+\beta$ :

- decreases up to $\Delta\left(\theta_{0}\right)$ on $\Delta_{0}<\beta<\Delta\left(\theta_{0}\right)-\theta_{0}$,

- increases from $\Delta\left(\theta_{0}\right)$ to infinity on $\beta \geq \Delta\left(\theta_{0}\right)-\theta_{0}$ (if $\theta_{0}$ is finite).

Thus $\theta^{p}(\beta)+\beta$ is non-monotonic implying a non-trivial comparison between the equilibria with known bias and unknown bias. We then obtain:

Proposition 3 There exists a non-empty interval $\left(\beta_{0}, \beta_{1}\right)$ such that $z^{u}-\beta>\theta^{p}(\beta)$ if and only if $\beta \in\left(\beta_{0}, \beta_{1}\right)$, where $\Delta_{0} \leq \beta_{0}<\beta_{1}$ (with $\beta_{1}=+\infty$ if $\left.\theta_{0}=-\infty\right)$.

Proof. To prove the result it suffices to show that $z^{u}>\min _{\beta}\left(\theta^{p}(\beta)+\beta\right)=\Delta^{p}\left(\theta_{0}\right)$. Suppose that $\Delta_{0}>z^{u}>z_{0}$. Notice that

$$
0=E\left[\theta \mid s \in\{\emptyset\} \cup\left(z_{0}-\beta, 0\right)\right]>E\left[\theta \mid s \in\{\emptyset\} \cup\left(z_{0}-\bar{\beta}, 0\right)\right]
$$

which implies that $z_{0}>\theta_{0}+\bar{\beta}$. Moreover

$$
\Delta^{u}\left(z_{0}\right)>\frac{\Delta_{0}}{1+\frac{p^{e}}{1-p^{e}} \max \left\{F(0)-F\left(z_{0}-\bar{\beta}\right) ; 0\right\}}=\Delta^{p}\left(z_{0}-\bar{\beta}\right)>\Delta^{p}\left(\theta_{0}\right) .
$$

Hence $z^{u}=\Delta^{u}\left(z^{u}\right)>\Delta^{u}\left(z_{0}\right)>\Delta^{p}\left(\theta_{0}\right)$.

A similar reasoning shows that when $z^{u}=z_{0}>\Delta^{u}\left(z_{0}\right)$, we have $z_{0}>\Delta^{p}\left(\theta_{0}\right)$.

If $\theta_{0}=-\infty$, then $\theta^{p}(\beta)+\beta$ declines and is smaller than $z^{u}$ for $\beta>\beta_{0}$. If $\theta_{0}$ is finite, then $\theta^{p}+\beta$ reaches a minimum $\Delta\left(\Theta_{N R}^{0}\right)$ then increases which yields the threshold $\beta_{1}$.

The surprising feature is that it is not always the case that transparency leads to more revelation of information. To discuss this issue further let us focus on the case in which $p^{e}$ is not too large 
so that $\theta_{0}=z_{0}=-\infty$ and only pure strategy equilibria exist. In this case, transparency reduces transmission of information for large realizations of the bias $\beta>\beta_{0}$. Thus the overall effect of transparency of the bias on the principal's welfare depends on the distribution of the bias.

Now suppose that the distribution of $\beta$ increases according to second order stochastic dominance. Then $E_{\beta}[\max \{F(0)-F(z-\beta) ; 0\}]$ increases so that $\Delta^{u}(z)$ move downward implying that $z^{u}$ decreases. Thus the agent will lie more at all levels of $\beta$ when the bias is not transparent and the distribution is shifted toward more bias. However at the same time the probability that $\beta>\beta_{0}$ increases making it more likely that transparency leads to less reporting. The overall effect is thus again ambiguous and depends on the mass of types of expert around $\beta=\beta_{0}$.

Notice that when the bias is not fully transparent, a procedure that succeeds in separating the most biased experts from others would raise $\Delta^{u}(z)$ for the remaining types and thus enhanced the information revealed by less biased experts. This can be achieved if the procedure succeed in revealing that the bias is above some level, or excluding such types. As, in practice, most of the experts with the highest expertise are those with the highest conflicts of interests, the decision of the FDA to avoid experts with conflicts of interests more important than some upper bound to participate in advisory committees seems to go in this way. But notice that this entails a cost, either by giving up some expertise or by increasing the extent of misreporting by these highly biased experts.

\section{$6 \quad$ Multiple experts}

So far we focused on the case of a single expert, but many expertise process can rely on several experts. Consulting a second expert allows the principal to obtain more information since this generates additional information when the first expert is not decisive, but it also affects the behavior of the first expert.

We examine these issues by considering the situation where two experts may intervene. Each expert may learn the value of $\theta$ or not, thus their signal is the same when they are both informed. However, they receive this information with independent probabilities. As before we say the expert/the report is decisive when the report is informative about $\theta$. For conciseness we assume that experts differs only by their bias with $\beta_{1}<\beta_{2} \cdot{ }^{11}$ Both are common knowledge. To simplify the

\footnotetext{
${ }^{11}$ The extension to different characteristics is starightforward and brings no new conceptual insight.
} 
analysis further, we assume first that truthtelling is never an equilibrium if there is only one expert $\left(\beta_{i}>\Delta_{o}\right)$. We also focus on situations where the principal always chooses $q=1$ if no expert sends a report. This is the case if $v_{i}(-\infty) \geq 0$ for both $i$, which we assume here. We finally assume that assumption $A$ holds. To summarize we assume here

Assumption $B: \beta_{2}>\beta_{1}>\Delta_{0} ;\left|\theta_{0}\right|$ is large or infinite, assumption $A$ holds.

Whether the presence of the second expert foster or imped information revelation by the first expert depends on the precise nature of the interaction and the information used by the market to update the beliefs on the level of expertise of each expert. Assuming as above that the principal is able to transmit to the market whether she has received decisive report or not, it remains the question of whether market knows which reports are decisive and which are not. In other words we should contrast the situation where the market receives information on each expert independnetly or whether he receives only collective information. The reason is that while reputation effects are not affected in the former case, pooling the information on expert may create some free-rider problem and dilutes the reputation effect. We thus examines the two cases.

\subsection{Full transparency and the disciplinary effect}

First, assume that the market observes whether the report of expert $i=1,2$ is decisive or not. The following analysis assumes that experts generate reports simultaneously. This amounts to say that the identity of the experts is publicly known at the beginning of the process, and that the principal declares at the end of the process whether a report is decisive, but this is not disclosed until the decision is announced.

It is immediate that the expert $i$ 's strategy is again a set $\Theta^{i}=\left(\theta_{i}, 0\right)$ of non-reported values. We set $\theta_{i}=0$ if the expert report truthfully $\left(\Theta^{i}=\varnothing\right)$. Morevover since all information is public, the reputation of an expert is not directly affected by the behavior of the other expert. The expert $i$ receives a reputation gain $\Delta_{i}=\Delta^{p}\left(\theta_{i}\right)$ by reporting the signal where the function is the same as in section 4.1 but indexed by the expert's identity.

Consider an expert $i$ with $\beta_{i}>0$ learning that $\theta<0$. Expert $i$ can anticipate the behavior of

the other expert and thus knows that the signal will be reported if it is below $\theta_{j}^{p}$. He thus report $\theta$ whenever

$$
\Delta_{i} \geq\left(1-p^{e} \mathbf{1}_{\theta \leq \theta_{j}}\right)\left(\theta+\beta_{i}\right)
$$


where $\mathbf{1}_{\theta \leq \theta_{j}}$ is an indicator that takes value 1 when the other expert reports the value $\theta$.

First notice that truthful reporting by one expert cannot be an equilibrium since by assumption $\beta_{i}>\Delta_{0}$. However it may be the case that both experts reveals truthfully which occurs when

$$
\Delta_{0} \geq\left(1-p^{e}\right) \beta_{i} \text { for } i=1,2 \text { and } j \neq i
$$

Thus the information revealed by each expert may increase when both are acting simultaneously. The reason is that transmitting information is less costly since the information may not be used. This increases the relative weight of reputation in the objective of each expert.

More generally let us define $\theta_{i}^{c}$ as the solution of

$$
\theta_{i}^{c}=\sup \left\{\theta \mid \theta \leq 0 \text { and } \Delta^{p}(\theta)-\left(1-p^{e}\right)\left(\theta+\beta_{i}\right) \geq 0\right\}
$$

Remind that the equilibrium threshold if there is only expert $i$ is $\theta_{i}^{p}$ solution of $\Delta^{p}\left(\theta_{i}^{p}\right)-\theta_{i}^{p}=\beta_{i}$. The value $\theta_{i}^{c}$ is the equilibrium threshold if the other expert reports truthfully, and it is larger than the former, $\theta_{i}^{c}>\theta_{i}^{p}$.

Given that $\beta_{1}<\beta_{2}$ we have $\theta_{1}^{p}>\theta_{2}^{p}$ and $\theta_{1}^{c} \geq \theta_{2}^{c}$. The equilibrium behavior of expert $i$ is then solution of

$$
\left\{\begin{array}{c}
\theta_{i}=\theta_{i}^{p} \text { if } \theta_{j} \leq \theta_{i}^{p} \\
\theta_{i}=\theta_{j} \text { if } \theta_{i}^{p} \leq \theta_{j} \leq \theta_{i}^{c} \\
\theta_{i}=\theta_{i}^{c} \text { if } \theta_{i}^{c} \leq \theta_{j} .
\end{array}\right.
$$

We then obtain the following equilibrium characterization:

Proposition 4 Assume $B$ and full transparency. The equilibrium with simultaneous expertise is characterized by

i) $\left(\theta_{1}^{s}, \theta_{2}^{s}\right)=\left(\theta_{1}^{p}, \theta_{2}^{c}\right)$ if $\theta_{1}^{p} \geq \theta_{2}^{c}$;

ii) $\theta_{1}^{p} \leq \theta_{1}^{s}=\theta_{2}^{s} \leq \theta_{2}^{c}$ if $\theta_{1}^{p}<\theta_{2}^{c}$.

Each expert reveals more information than if he is the sole expert (and strictly for at least one expert).

Proof. First we have $\theta_{1} \leq \theta_{2}$ since the reverse inequality would imply $\theta_{1}^{p}=\theta_{1}>\theta_{2}=\theta_{2}^{c}$ which is not possible. An equilibrium with $\theta_{1}<\theta_{2}$ must verify $\theta_{1}=\theta_{1}^{c}$ and $\theta_{2}=\theta_{2}^{p}$ which is only possible if $\theta_{1}^{c}<\theta_{2}^{p}$. An equilibrium with $\theta_{1}=\theta_{2}$ requires $\theta_{1}^{p} \leq \theta_{1}=\theta_{2}=\min \left(\theta_{1}^{c}, \theta_{2}^{c}\right)$ which is only possible if $\theta_{2}^{p} \leq \theta_{1}^{c}$. 
The last point follows from $\theta_{i}^{c}>\theta_{i}^{p}$.

The intuition developed abover thus extends. Using several experts in the context of full tranapency on each expert contribution enhances the incentive power of reputation effects by reducing the perceived cost of misreporting.

An immediate consequence is that the value of the second expert is larger than the value of the additional information provided. Indeed the value of the second expert is $V_{P}^{s}-V_{P}^{1}$ where (using $\left.\theta_{2}^{s} \leq \theta_{1}^{s}\right)$

$$
V_{P}^{1}=p^{e}\left(-\int_{+\infty}^{\theta_{1}^{p}} \theta f(\theta) d \theta\right) ; V_{P}^{s}=p^{e}\left(-\int_{+\infty}^{\theta_{1}^{s}} \theta f(\theta) d \theta\right)+\left(1-p^{e}\right) p^{e}\left(-\int_{+\infty}^{\theta_{2}^{s}} \theta f(\theta) d \theta\right)
$$

We can then decompose that value of the second expert into two parts

$$
V_{P}^{s}-V_{P}^{1}=\left(1-p^{e}\right) p^{e}\left(-\int_{+\infty}^{\theta_{2}^{s}} \theta f(\theta) d \theta\right)+p^{e}\left(-\int_{\theta_{1}^{p}}^{\theta_{1}^{s}} \theta f(\theta) d \theta\right)
$$

The first term is the value of the information provided by the second expert. The second term capture the value attached to enhanced incentives for the first expet and it is positive whenenever the second expert is not too biased so that $\theta_{2}^{c}>\theta_{1}^{p}$.

When hiring an expert is costly, the principal may decide to consult sequentially. Then the principal asks the report of expert $i$, and only if this is not decisive the expertise of expert $j$. It is then immediate that the second expert follows the strategy $\theta_{j}=\theta_{j}^{p}$ since he knows that his report is the only relevant one when he is consulted. Thus he reports less than in the simultaneous consultation case. Given that best replies are monotonic, this conclusion extends to the other expert. We then have

Corollary 4 A simultaneous consultation of experts leads to (weakly) more information revealed than a sequential consultation.

Proof. If expert 1 is consulted first, then we have $\theta_{i}=\theta_{i}^{p}$ for both experts. If expert 2 is consulted first we have $\theta_{1}=\theta_{1}^{p}$ and $\theta_{2}=\min \left(\theta_{1}^{p}, \theta_{2}^{c}\right)$. In all cases the level $\theta_{i}$ is smaller than in the simultaneous case for both experts.

The consequence of this corollary is that when expertise is costly, there is some ambiguity on the optimal timing. Indeed a sequential consultation leads to less information but saves on expertise 
costs. More precisely let $w$ be the cost of an expertise assumed to be fixed an independent of the expert. ${ }^{12}$ Let $V_{P}^{i j}$ be the gross welfare under a sequential procedure starting with expert $i$. Then we have

$$
V_{p}^{12}=V_{P}^{1}+\left(1-p^{e}\right) p^{e}\left(-\int_{+\infty}^{\theta_{2}^{p}} \theta f(\theta) d \theta\right) \leq V_{p}^{21}=V_{P}^{1}+\left(1-p^{e}\right) p^{e}\left(-\int_{+\infty}^{\min \left(\theta_{1}^{p}, \theta_{2}^{c}\right)} \theta f(\theta) d \theta\right) \leq V_{P}^{s}
$$

Denoting $W_{P}^{i j}=w\left(2-p^{e}+p^{e}\left(F(0)-F\left(\theta_{i}^{i j}\right)\right)\right)$ the expected cost we also have $W_{p}^{s}=2 w$ and

$$
\begin{aligned}
W^{21}-W_{p}^{12} & =w p^{e}\left(F\left(\theta_{1}^{p}\right)-F\left(\min \left(\theta_{1}^{p}, \theta_{2}^{c}\right)\right)\right) \geq 0 \\
W_{p}^{s}-W_{P}^{21} & =w p^{e}\left(1-F(0)+F\left(\min \left(\theta_{1}^{p}, \theta_{2}^{c}\right)\right)\right)>0
\end{aligned}
$$

In a sequential timing, more information is revealed if the most biased expert (expert 2) is consulted first, as he is constrained by the next expertise. In terms of cost, the order is reversed. Simultaneous consultation is the most costly as the cost is spend twice, while the less costly procedure is when expert 1 is consulted first as this maximizes the chance of obtaining the information with only one expert.

One conclusion is however that when the difference between the two experts is not too large, we will have $\theta_{1}^{p}<\theta_{2}^{c}$. Then $W_{p}^{12}=W_{p}^{21}$ since a sequential consultation starting with expert 2 leads to $\theta_{2}=\theta_{1}^{p}$ and thus the same probability of obtaining the information in the first round than the reverse timing. Moreover $V_{p}^{12}<V_{p}^{21}$ whenever $\theta_{1}^{p}>\theta_{2}^{p}$. It follows that when experts' preferences are not too far apart, the optimal sequential timing is to start with the most biased expert (expert $2)$.

Another conclusion from the analysis is that the sequential procedure with the expert 2 consulted first may be subject to a time inconsistency issue. Indeed the principal may wish to commit to a second expertise in order to raises the incentives of the first expert, but ex-post she will be tempted to save the cost and renege on the commitment if the cost is larger than the value of the additional information. To see that notice that the value of the second expert ex-ante is (assuming $\theta_{2}^{c}>\theta_{1}^{p}$ )

$$
V_{P}^{21}-V_{P}^{1}-\left(W_{p}^{21}-w\right)=\left(1-p^{e}\right) p^{e}\left(-\int_{+\infty}^{\theta_{1}^{p}} \theta f(\theta) d \theta\right)-
$$

\footnotetext{
${ }^{12}$ In many public expertise procedures, the cost is governed by rigid administrative rules.
} 


\subsection{Ambiguity on the decisive expert and dilution of incentives}

Things may be quite different whenever the market is not fully informed about the contribution of each expert. Indeed, when the market information is coarse, it may fail to attribute the credit of a decisive report to the correct expert with the result of a dilution of the reputation effect between the two experts. Then reputation become a collective good for the experts, raising the issue of moral hazard in teams and poor incentives (Holsmtrom, 1982). To see that we extend the model by assuming that while the principal reveals whether she had access to a decisive report, she does not reveal ex-post the identity of the decisive expert. This would be the case for procedures that report the names of the expert and where the decision of the principal is "motivated", meaning that the principal attaches to the decision the reasoning and elements that justify it. In this case, one can see whether some specialized information was used but may not see which expert provided this information.

Thus the market observes the decision and whether some expert were decisive. ${ }^{13}$ Since the market cannot infer which expert was decisive, the information used by the market to assess reputation becomes a joint production for the two experts. Now expert $i$, anticipating that expert $j$ reveals $\theta<\theta_{j}$, chooses to announce his information $\theta<0$ if

$$
\left(1-p^{e} \mathbf{1}_{\theta \leq \theta_{j}}\right)\left(\Delta_{i}-\theta-\beta_{i}\right) \geq 0
$$

where $\Delta_{i}=\Delta^{m}\left(\theta_{i}, \theta_{j}\right)$ is the reputation gain of expert $i$ when the market knows that one expert reported a negative value of $\theta$. This writes as:

$$
\Delta^{m}\left(\theta_{i}, \theta_{j}\right)=\frac{\pi\left(\bar{p} F\left(\theta_{i}\right)+p^{e} F\left(\theta_{j}\right)-\bar{p} p^{e} \min \left(F\left(\theta_{1}\right), F\left(\theta_{2}\right)\right)\right)}{p^{e} F\left(\theta_{i}\right)+p^{e} F\left(\theta_{j}\right)-\left(p^{e}\right)^{2} \min \left(F\left(\theta_{1}\right), F\left(\theta_{2}\right)\right)}-\frac{\pi\left(1-\bar{p}+\bar{p}\left(F(0)-F\left(\theta_{i}\right)\right)\right)}{1-p^{e}+p^{e}\left(F(0)-F\left(\theta_{i}\right)\right)}
$$

Since only the joint report is relevant for the expert, his report matters only when $\theta$ is not reported by the other expert, which occurs with probability $1-p^{e} \mathbf{1}_{\theta \leq \theta_{j}}$. Conditional on no report by the other expert, the incentives to report are unchanged compared to the case with one expert. But the reputation gain $\Delta_{i}$ is affected. The effect on equilibrium behaviors then depends on the comparison between the reputation gain with one and two experts.

Since the market is not able to infer which expert produced the decisive report, the individual reputation gain should be smaller when there is a second expert. Indeed we show below that

\footnotetext{
${ }^{13}$ We exmaine in our 2010 working paper the case where only the decision is observed.
} 
$\Delta^{m}\left(\theta_{i}, \theta_{j}\right)<\Delta^{p}\left(\theta_{i}\right)$. The meaning of this result is that, in all pure strategy equilibria, hiring a second expert reduces the first expert's incentives to reveal his information when it is unfavorable. We thus obtain that

Proposition 5 Asumme $B$ and the identity of the decisive experts is not public, then each expert reveals strictly less information when there are two experts than if he is the sole expert..

Proof. It suffices to show that $\Delta^{m}\left(\theta_{i}, \theta_{j}\right)<\Delta^{p}\left(\theta_{i}\right)$ or

$$
\frac{\pi\left(\bar{p} F\left(\theta_{i}\right)+p^{e} F\left(\theta_{j}\right)-\bar{p} p^{e} \min \left(F\left(\theta_{1}\right), F\left(\theta_{2}\right)\right)\right)}{p^{e} F\left(\theta_{i}\right)+p^{e} F\left(\theta_{j}\right)-\left(p^{e}\right)^{2} \min \left(F\left(\theta_{1}\right), F\left(\theta_{2}\right)\right)}<\frac{\pi \bar{p}}{p^{e}}
$$

which holds provided that $p^{e}<\bar{p}$. Then assumption $A$ ensures that on $\theta \geq \theta_{i}^{p}: \Delta^{m}\left(\theta, \theta_{j}\right)-\theta-\beta_{i}<$ $\Delta^{p}(\theta)-\theta-\beta_{i} \leq 0$. Hence in any equilibrium of the multiple expert game we have $\theta_{i}>\theta_{i}^{p}$.

Thus consulting two experts raises the number of sources of information, which raises the chance to learn that the status quo is not the correct decision, but il also undermine the incentives of experts by diluting the reputtaion effects between the two experts. The value of the second expert may then not be positive. Indeed denoting $\theta_{i}^{m}<\theta_{i}^{p}$ the threshold levels, the value $V_{P}^{m}$ for the principal is

$$
V_{P}^{m}=V_{P}^{1}+\left(1-p^{e}\right) p^{e}\left(-\int_{+\infty}^{\theta_{2}^{m}} \theta f(\theta) d \theta\right)-p^{e}\left(-\int_{\theta_{1}^{m}}^{\theta_{1}^{p}} \theta f(\theta) d \theta\right) .
$$

The second bracket term is positive so that the value of the second expert is smaller than the value of the additional information provided, and can even be negative.

Considering the timing of the procedure, notice that provided that the market information is not altered, a sequential consultation of experts would result in the same strategies by experts and thus would not alter the level of informtaion transmited (but would be less costly). However it most likely that a sequential consultation would alter the nature of the market information and of the reputation effect. For example if the names of experts and the order of consultation are public information, then the sequential timing reveals the identity of the decisive expert (they can be only one). Even if the order is not public, when he is decisive, the first expert benefits from a strong reputation gain $\Delta^{p}\left(\theta_{i}\right)$ since he is known to be the sole expert. This suggests that a sequential timing should be preferred in this case. 


\section{Conclusion}

This paper examines the efficiency of (hard) information transmission between some biased experts and a decision maker, focusing on reputation mechanisms for experts. Biased experts have an incentive to conceal unfavoured pieces of information. There is a complex interaction between reputation effects and these incentives, because the reputation effects are reinforced when the incentives to conceal are weakened.

In this context, hiring experts with a lower bias in favour of the status quo improves the efficiency of the decision. Moreover the amount of information revealed by experts increases as the information of the market on the details of experts' contributions increases (we develop this point at more length for the single expert case in our working paper). But an interesting result is that it is not always the case that transparency on the experts' bias leads to more revelation of information.

Finally we show that the impact of consulting another expert on the amount of information concealed by the expert depends on the information affecting reputation. The incentives to reveal information are strenghthened when information allows the market to identify each expert's contribution, so that reputation effects are independent between the experts. But when the market observes a coarser information, reputation effects are interdependent, in which case the incentives to reveal information are weakened. The optimal timing of consultation depends also on the nature of the information revealed to the market under the different timing.

When the experts' reports are public, the presence of another expert induces an expert to report more truthfully. However, this result is reversed in the case of secret reports. The intuitive result that hiring an expert constrains other experts to reveal the truth is therefore only true when there is public disclosure of reports. The optimal timing with public reports depends on the costs of expertise while a sequential timing is optimal with secret reports.

Notice that the sequential policy raises time consistency issues. For instance, in the case of public reports, the principal may be tempted to announce that she will consult a second expert and then renege on the promise and not consult the second expert. The reason is that committing to consult raises the incentives of the first expert and thus has a value larger than just the value of

the information provided by the second expert. Similarly, in the case of secret reports, it may be optimal to commit not to consult the other expert, but then to renege on the promise. The reason 
is again that such a commitment allows to raise the incentive on the second expert.

One aspect that we left aside is that the bias itself may be affected by the information disclosed This is the case when the expert anticipate some future rents from the relationship with an interested party and fear that a negative report will trigger some form of retaliation by this party. Also the expert may be tempted to misreport if this raises his future prospect with this party. In other words reputation effects on quality of experts may conflict with other forms of reputation concerns. ${ }^{14}$

\section{Appendix}

Reputation gain . We will first compute the gain of reputation when when the expert announces an informative report for $\theta \in \Theta=\left(\theta^{p}, 0\right)$ :

$$
\begin{gathered}
\operatorname{Pr}(\bar{p} \mid r=\emptyset)=\frac{\pi(1-\bar{p}+\bar{p} \operatorname{Pr}(\Theta))}{1-p^{e}+p^{e} \operatorname{Pr}(\Theta)} \\
\operatorname{Pr}(\bar{p} \mid r=\theta)=\frac{\pi \bar{p}}{p^{e}}
\end{gathered}
$$

This gives

$$
\Delta^{p}(\theta)=\frac{\Delta_{0}}{1+\frac{p^{e}}{1-p^{e}}(F(0)-F(\theta))} .
$$

Quasi-concavity of $(\theta+\beta) / \Delta^{p}(\theta)-1$. Let

$$
g(\theta)=(\theta+\beta)\left(1+\frac{p^{e}}{1-p^{e}}(F(0)-F(\theta))\right)
$$

Then $(\theta+\beta) / \Delta^{p}(\theta)-1=g(\theta) / \Delta_{0}-1$. It suffice to show that $g$ is quasi-concave. The first and second derivatives are

$$
\begin{aligned}
g^{\prime}(\theta) & =\left(1+\frac{p^{e}}{1-p^{e}}(F(0)-F(\theta)-(\theta+\beta) f(\theta))\right) \\
g^{\prime \prime}(\theta) & =\frac{p^{e}}{1-p^{e}}\left(-2 f(\theta)-(\theta+\beta) f^{\prime}(\theta)\right)
\end{aligned}
$$

Notice tht $g^{\prime \prime}$ is negative if $f^{\prime} \geq 0$. Suppose that $g^{\prime}(\theta) \leq 0$ and $f^{\prime}(\theta)<0$. Then $\frac{F(0)-F(\theta)}{f(\theta)}<\theta+\beta$ and

$$
2 f(\theta)-(\theta+\beta) f^{\prime}(\theta)<-2 f(\theta)-\frac{F(0)-F(\theta)}{f(\theta)} f^{\prime}(\theta) .
$$

\footnotetext{
${ }^{14}$ In our model one could think for exemple that the expert cares about signalling his bias $\beta$ to a third party by not reporting.
} 
But under assumption $A, \quad f(\theta)+\frac{F(0)-F(\theta)}{f(\theta)} f^{\prime}(\theta) \geq 0$.Thus

$$
g^{\prime \prime}(\theta)<-2 f(\theta)-\frac{F(0)-F(\theta)}{f(\theta)} f^{\prime}(\theta)<-f(\theta)<0 .
$$

This implies that $g$ is decreasing on an interval $\left(\theta^{\prime}, 0\right)$ and increasing on $\left(-\beta, \theta^{\prime}\right)$.

Moreover $g(-\beta) / \Delta_{0}-1=-1$ and $g(0)=\beta / \Delta_{0}-1>0$.

\section{References}

[1] Angell, M., March 10, 2005, "What ails the FDA? Payola," Boston Globe.

[2] Beniers, K.J. and O.H. Swank, 2004, "On the Composition of Committees," Journal of Law, Economics, and Organization, 20, 353-378.

[3] Bourjade, S. and B. Jullien, 2010, "Expertise and Bias in Decision Making," Working Paper, Toulouse Business School.

[4] Bruce, B., 2002, "Stock Analysts: Experts on Whose Behalf," The Journal of Psychology and Financial Markets, 3, 198-201.

[5] Cauchon, D., September 25, 2000, "Number of drug experts available is limited: Many waivers granted for those who have conflicts of interest," USA Today.

[6] Crawford, V. and J. Sobel, 1982, "Strategic Information Transmission," Econometrica, 50(6), 1431-1452.

[7] Dewatripont, M. and J. Tirole, 1999, "Advocates," Journal of Political Economy, 107, 1-39.

[8] Dur, R. and O. Swank, 2005, "Producing and Manipulating Information," The Economic Journal, 115, 185-199.

[9] Fudenberg, D., D. Kreps and D. Levine, 1988, "On the Robustness of Equilibrium Refinements," Journal of Economic Theory, 44, 354-380.

[10] Grossman, S., 1981, "The informational role of warranties and private disclosure about product quality," Journal of Law and Economics, 24, 461-483.

[11] Gromb, D. and D. Martimort, 2007, "Collusion and the Organization of Delegated Expertise," Journal of Economic Theory, 137, 271-299. 
[12] Harris, G., July 24, 2006, "F.D.A. rules will regulate experts' ties to drug makers," New York Times.

[13] Henderson, D., April 21, 2006, "No end to FDA disclosure debate," Boston Globe.

[14] Henry, E., 2009, "Strategic Disclosure of Research Results: The Cost of Proving Your Honesty," The Economic Journal, 119, 1036-1064.

[15] Holmstrom, B., 1982, "Moral Hazard in Teams," The Bell Journal of Economics, 13, 324-340

[16] Kofman, F., J. Lawaree, 1993, "Collusion in hierarchical agency," Econometrica, 61, 629-656.

[17] Krishna, V. and J. Morgan, 2001, "A model of expertise," Quarterly Journal of Economics, $116,747-775$.

[18] Kuhn, K-U., 2002, "Reforming European Merger Review: Targeting Problem Areas in Policy Outcomes," Journal of Industry, Competition and Trade, 2, 311-364.

[19] Laffont, J.-J. and J. Tirole, 1991, "The Politics of Government Decision-Making: a Theory of Regulatory Capture," Quarterly Journal of Economics, 106, 1089-1127.

[20] Li, M., 2009, "Combining Expert Opinions," Working Paper, Concordia University.

[21] Milgrom, P., 1981, "Good news and bad news: Representation theorems and applications," Bell Journal of Economics, 12, 380-391.

[22] Milgrom, P. and J. Roberts, 1986, "Relying on the Information of Interested Parties," Rand Journal of Economics, 17, 18-32.

[23] Morgan, J. and P. Stocken, 2003, "An Analysis of Stock Recommendations," Rand Journal of Economics, 34(1), 183-203.

[24] Morris, S., 2001, "Political Correctness," Journal of Political Economy, 109, 231-265.

[25] Ottaviani, M. and P.N. Sorensen, 2001, "Information aggregation in debate: who should speak first?," Journal of Public Economics, 81, 393-421.

[26] Ottaviani, M. and P. N. Sorensen, 2006a, "Professional Advice: The Theory of Reputational Cheap Talk," Journal of Economic Theory, 126, 120-142. 
[27] Ottaviani, M. and P. N. Sorensen, 2006b, "Reputational Cheap Talk," RAND Journal of Economics, Spring 2006, 37, 155-175.

[28] Prendergast, C., 1993, "A theory of yes men," American Economic Review, 83, 757-770

[29] Rey, P., 2003, "Towards a Theory of Competition Policy," in Advances in Economics and Econometrics: Theory and Applications - Eight World Congress, M. Dewatripont, L. P. Hansen and S. J. Turnovsky (eds.), Cambridge University Press.

[30] Roller, L.-H., Stennek, J. and F. Verboven, 2001, "Efficiency Gains from Mergers," European Economy, 5, 31-127.

[31] Shin, H.S., 1994, "News management and the value of firms," Rand Journal of Economics, 25, $58-71$.

[32] Shin, H.S., 1998, "Adversarial and Inquisitorial Procedures in Arbitration," Rand Journal of Economics, 29, 378-405.

[33] Shin, H.S., 2003, "Disclosures and Asset Returns," Econometrica, 71(1), 105-133.

[34] Sobel, J., 1985, “A Theory of Credibility,” Review of Economics Studies 52, 557-573.

[35] Suurmond, G., Swank, O.H. and B. Visser, 2004, "On the bad reputation of Reputational Concerns," Journal of Public Economics, 88, 2817-2838.

[36] Wolinsky, A., 2003, "Information transmission when the sender's preferences are uncertain," Games and Economic Behavior, 42, 319-326. 